\title{
Azithromycin alleviates the severity of rheumatoid arthritis via targeting UPR component GRP78
}

\author{
Yongli Zhang ${ }^{1}$, Luna $\mathrm{Ge}^{2}$, Guanhua Song ${ }^{1}$, Ruojia Zhang ${ }^{1}$, Shufeng Li $^{2}$, Haojun Shi $^{3}$, \\ Hongchang Zhang ${ }^{1}$, Yi Li ${ }^{4}$, Jihong Pan $^{2}$, Lin $\mathrm{Wang}^{2}$, and Jinxiang $\mathrm{Han}^{2}$ \\ ${ }^{1}$ Shandong First Medical University \\ ${ }^{2}$ First Affiliated Hospital of Shandong First Medical University \\ ${ }^{3}$ Henan University of Traditional Chinese Medicine \\ ${ }^{4}$ Shandong Provincial Hospital
}

January 20, 2021

\begin{abstract}
Background and Purpose: Azithromycin (AZM) is a macrolide antibiotic with well-described anti-inflammatory properties. This study aimed to substantiate its treatment potential in rheumatoid arthritis (RA). Experimental Approach: Gene expression profiles were collected by RNA-sequencing and the effects of AZM were assessed in functional assays. In vitro and vivo assays for examining the blockade of glucose-regulated protein 78 (GRP78) actions by AZM: assays for defining the anti-inflammatory activity of AZM using fibroblast-like synoviocytes (FLSs) from RA patients as well as collagen-induced arthritis (CIA) in DBA/1 mice. Identification and characterization of the binding of AZM to GRP78 using drug affability responsive target stability assay, proteomics and cellular thermal shift assay. Detect AZM inhibition of GRP78 and dependence of AZM's antiarthritis activity on GRP78. Key Results: AZM reduced pro-inflammatory factor production, cell migration, invasion and chemo-attractive potential, enhanced apoptosis, thereby reducing the deleterious inflammatory response of RA FLSs in vitro. AZM ameliorated the severity of CIA lesions. Transcriptional analyses implied that AZM treatment causes impairments in signaling cascades associated with cholesterol and lipid biosynthetic process. GRP78 was isolated as a novel target of AZM. AZM-mediated activation of unfolded protein response (UPR) via inhibiting GRP78 activity is required not only for inducing the expression of $\mathrm{C}$ /EBP-homologous protein (CHOP), but also for activation of sterol-regulatory element binding protein (SREBP) and its targeted genes involved in cholesterol and lipid biosynthetic process. Further, deletion of GRP78 abolished AZM's anti-arthritis activity. Conclusion and Implications: These findings confirmed that AZM is an anti-arthritis therapeutic drug for RA treatment.
\end{abstract}

\section{Title}

Azithromycin alleviates the severity of rheumatoid arthritis via targeting UPR component GRP78

Running title

Azithromycin relieves the severity of rheumatoid arthritis

Authors

Yongli Zhang, ${ }^{1, \#}$, Luna Ge $\mathrm{Ge}^{1,2, \#}$, Guanhua Song ${ }^{3}$, Ruojia Zhang ${ }^{1}$, Shufeng $\mathrm{Li}^{4}$, Haojun $\mathrm{Shi}^{5}$, Hongchang Zhang ${ }^{1}$, Yi Li ${ }^{6}$, Jihong Pan $^{1,2^{*}}$, Lin Wang ${ }^{1,2^{*}}$, Jinxiang $\operatorname{Han}^{1,2^{*}}$

\#These two authors contributed equally to this work.

\section{Affiliations}


1. Biomedical Sciences College of Shandong First Medical University \& Shandong Medicinal Biotechnology Centre, Key lab for Biotech-Drugs of National Health Commission, Key Lab for Rare \& Uncommon Diseases of Shandong Province, Shandong Medicinal Biotechnology Centre, Jinan, China; 2. Department of Rheumatology and Autoimmunology, The First Affiliated Hospital of Shandong First Medical University, Jinan, China; 3. Institute of Basic Medicine, Shandong First Medical University \& Shandong Academy of Medical Sciences, Jinan, China; 4. Department of Orthopedic Surgery, The First Affiliated Hospital of Shandong First Medical University, Jinan, China; 5. The second clinical medical college, Henan University of Chinese Medicine, Zhengzhou, China. 6. Department of Joint Surgery, Shandong Provincial Hospital Affiliated to Shandong University, Jinan, China.

\section{*Corresponding author:}

*Lin Wang Ph. D., Department of Rheumatology and Autoimmunology, The First Affiliated Hospital of Shandong First Medical University; Biomedical Sciences College \& Shandong Medicinal Biotechnology Centre, Key lab for Biotech-Drugs of National Health Commission, Key Lab for Rare \& Uncommon Diseases of Shandong Province, Shandong First Medical University \& Shandong Academy of Medical Sciences, \#18877, Jingshi Road, Jinan, 250062 P.R. China.

Tel: $+86-531-82629328$

E-mail: kecheng1216@163.com

*Jinxiang Han Ph. D., Department of Rheumatology and Autoimmunology, The First Affiliated Hospital of Shandong First Medical University; Biomedical Sciences College \& Shandong Medicinal Biotechnology Centre, Key lab for Biotech-Drugs of National Health Commission, Key Lab for Rare \& Uncommon Diseases of Shandong Province, Shandong First Medical University \& Shandong Academy of Medical Sciences, \#18877, Jingshi Road, Jinan, 250062 P.R. China.

Tel: $+86-531-82629328$

E-mail: sams-h2016@163.com

\section{Word count}

5509 words (excluding figure legends and references)

\section{Acknowledgements}

None.

\section{Conflict of interest statement}

There are no conflicts of interest by any author.

\section{Data Availability Statement}

Data are available upon reasonable request. All data relevant to the study are included in the article or uploaded as supplementary information.

\section{Contributors}

LG, LW and JH designed the research. YZ, LF, GS, RZ, HS and HZ performed the research. SL and YL helped in sample collection. LG, JP, LW and JH analyzed the data. All the authors contributed to writing the paper.

\section{Funding}

This work was supported by National Natural Science Foundation of China (Grant No. 81772760, 82072850), The Shandong Taishan Scholarship (NO. tsqn20161076), The Innovation Project of Shandong Academy of Medical Sciences (2021), The Youth Innovation Technology Plan of Shandong University (2019KJK003) and Academic promotion programme of Shandong First Medical University (LJ001). 


\section{Patient consent for publication}

obtained.

\section{Ethics approval}

The studies were approved by the institutional review Board of Shandong research center for Medicinal Biotechnology.

\section{Abstract}

Background and Purpose: Azithromycin (AZM) is a macrolide antibiotic with well-described antiinflammatory properties. This study aimed to substantiate its treatment potential in rheumatoid arthritis (RA).

Experimental Approach: Gene expression profiles were collected by RNA-sequencing and the effects of AZM were assessed in functional assays. In vitro and vivo assays for examining the blockade of glucoseregulated protein 78 (GRP78) actions by AZM: assays for defining the anti-inflammatory activity of AZM using fibroblast-like synoviocytes (FLSs) from RA patients as well as collagen-induced arthritis (CIA) in DBA/1 mice. Identification and characterization of the binding of AZM to GRP78 using drug affability responsive target stability assay, proteomics and cellular thermal shift assay. Detect AZM inhibition of GRP78 and dependence of AZM's anti-arthritis activity on GRP78.

Key Results: AZM reduced pro-inflammatory factor production, cell migration, invasion and chemoattractive potential, enhanced apoptosis, thereby reducing the deleterious inflammatory response of RA FLSs in vitro . AZM ameliorated the severity of CIA lesions. Transcriptional analyses implied that AZM treatment causes impairments in signaling cascades associated with cholesterol and lipid biosynthetic process. GRP78 was isolated as a novel target of AZM. AZM-mediated activation of unfolded protein response (UPR) via inhibiting GRP78 activity is required not only for inducing the expression of C/EBP-homologous protein (CHOP), but also for activation of sterol-regulatory element binding protein (SREBP) and its targeted genes involved in cholesterol and lipid biosynthetic process. Further, deletion of GRP78 abolished AZM's anti-arthritis activity.

Conclusion and Implications: These findings confirmed that AZM is an anti-arthritis therapeutic drug for RA treatment.

\section{Keywords}

Rheumatoid arthritis, Azithromycin, Fibroblasts, UPR, GRP78

\section{Abbreviations}

RA, rheumatoid arthritis; FLSs, fibroblast-like synoviocytes; PBMC, peripheral blood mononuclear cell; AZM, azithromycin; ETC, etanercept; NF- $x$ B, nuclear factor kappa-B; IKK $\beta$, inhibitor kappa B kinase $\beta \cdot$ ER, endoplasmic reticulum; UPR, unfolded protein response; qRT-PCR, Quantitative real-time PCR; ELISA, Enzyme-linked immunosorbent assay; siRNA, Small interfering RNA; co-IP, Co-immunoprecipitation; DARTS, Drug Affinity Responsive Target Stability; MS, Mass spectrometry; CETSA, Cellular thermal shift assay; LPS, lipopolysaccharides; IL, interleukin; MMP, matrix metallopeptidase; TNF- $\alpha$, tumor necrosis factor; CXCL, C-X-C motif chemokine ligand; Tm, tunicamycin; Tg, thapsigargin.

\section{Main text}

\section{INTRODUCTION}

Dysregulated adaptive immunity can precede the clinical manifestation of affected joints in rheumatoid arthritis (RA) and the repeated activation of innate immunity can contribute to the hyper-inflammation and disease progression of RA (O'Neil \& Kaplan, 2019). Identification and characterization of novel, safer and more cost-effective drug, in particular antagonists with different inhibitory properties, are of great importance. Of the important cellular participants in the joints of RA, synovial lining fibroblasts [also 
called fibroblast-like synoviocytes (FLSs)] exhibit an aggressive and apoptosis resistant phenotype (Bartok \& Firestein, 2010) and produce excessive cytokines, small molecule mediators of inflammation(Weijun et al., 2018). and proteolytic enzymes (Ai et al., 2018). Therefore, inhibiting the ensuing inflammatory cycle of FLSs might improve clinical outcomes in RA patients.

Azithromycin (AZM) is a broad-spectrum antibiotic against respiratory, urogenital, dermal and other bacterial infections (Parnham, Haber, Giamarellos-Bourboulis, Perletti, \& Vos, 2014). It has recently received increasing attention because of additional effects on chronic inflammatory disorders such as diffuse panbronchiolits, bronchiolitis obliterans and rosacea (Spagnolo, Fabbri, \& Bush, 2013). The anti-inflammatory effects of AZM could be ascribed to prevent oxidative stress, cytokine productions, fibrosis occurrence and the consequent tissue destruction (Vanaudenaerde, Wuyts, Geudens, Dupont, \& Verleden, 2010). The immunomodulation of AZM is associated with the inhibition of nuclear factor kappa-B (NF- $x \mathrm{~B})$ mediators, inhibitor kappa B kinase $\beta$ (IKK $\beta$ ) (Haydar et al., 2019). Importantly, AZM could inhibit inflammation and liposomal enzyme release in arthritic rats (Liu, Pu, Li, Zhou, \& Wan, 2017), even though its exact effects and the molecular mechanism remain obscure (Nujić, Banjanac, Munić, Polančec, \& Eraković Haber, 2012). Importantly, fibroblasts, due to their wide distribution, have been proposed as a potential reservoir for AZM, slowly releasing or passing it to nearby phagocytes for transport to the site of infection (Cory et al., 2013; Ozsvari, Nuttall, Sotgia, \& Lisanti, 2018). While RA FLSs contribute greatly to the pathological progression of RA, characterizing the effects of AZM on RA FLSs would provide novel evidence for AZM in RA treatment (Zhang et al., 2015).

The endoplasmic reticulum (ER) is the site of biosynthesis for all secreted and membrane proteins and the accumulation of unfolded proteins in the ER leads to ER stress (Lu et al., 2020). Unfolded protein response (UPR) represents as an adaptive mechanism to keep homeostasis of ER in response to ER stress (Hetz \& Saxena, 2017). Especially, unlike other cell types, RA FLSs are resistant against ER stress-induced apoptosis (Rahmati, Moosavi, \& McDermott, 2018). As inflammation and the ER stress pathways are interconnected and concurrently regulated (Xu, Yang, Berezowska, Gao, \& Peng, 2019), a more effective approach being able to modify integrated biological outcomes by simultaneously targeting both ER stress and inflammation pathways can be of major therapeutic benefit (Reverendo, Mendes, Argüello, Gatti, \& Pierre, 2019). Herein, we provide genetic and biochemical evidence that AZM can serve as a promising approach to treat RA and identified GRP78-mediated UPR activation as a critical anti-arthritis function of AZM.

\section{MATERIAL AND METHODS}

\section{Patients}

RA synovial tissue was obtained from RA patients $(\mathrm{n}=12$, five males, seven females, aged 30-70 years old, mean age 50 years) during synovectomy or joint replacement surgery at the Shandong Provincial Hospital (Jinan, China). All patients met the diagnostic criteria for RA as revised by the American college of rheumatology in 1987. The majority of patients were treated with disease-modifying antirheumatic drugs (DMARDs), including methotrexate, and none were on biologics. Demographic data are provided in Online Supplementary Table 1. The study was approved by the Institutional Review Board of Shandong Medicinal Biotechonogy Center (SMBC17LL007) and was conducted according to the recommendations of the Declaration of Helsinki. All patients provided informed consent for participation in the study.

\section{Cell isolation and stimulation}

RA FLSs were obtained from the synovial tissues of RA as described previously (L. Wang et al., 2016) and cells were cultured in a medium of Dulbecco's modified Eagle's medium (DMEM) supplemented with 10\% fetal bovine serum (FBS) and 1\% antibiotics. RA FLSs between passages 4 and 7 were maintained for further experiments. Human peripheral blood mononuclear cell (PBMC) were isolated from venous blood of RA patients by density gradient centrifuging with Lympholite/H. The gradients were spun at $800 \mathrm{~g} \times 10$ minutes at $22{ }^{\circ} \mathrm{C}$. PBMC cells at the interface were removed, washed twice and resuspended in serum-free medium (RPMI 1640). The PBMC preparations were then ready for AZM treatment. AZM and Etanercept (ETC), dissolved in dimethyl sulphoxide at a concentration of $1 \mathrm{mM}$, was brought to the final concentration with 
complete medium. Before treatment with AZM and ETC, RA FLSs and PBMC were cultured overnight in medium containing 1\% fetal cells and subsequently stimulated with IL-1 $\beta$ (\#8900, Cell Signaling Technology, USA) and TNF- $\alpha$ (\#7321, Cell Signaling Technology, USA), or lipopolysaccharides (LPS, Sigma, St. Louis, MO, USA).

\section{Migration, invasion and tube formation assay}

The migration and invasion assay were performed as described before(Mierke, 2019). To examine the effect of AZM on tube formation, Matrigel $(10 \mathrm{mg} / \mathrm{mL}$, BD Biosciences, San Jose, CA, USA) was firstly plated in 96-well culture plates and allowed to polymerise at $37{ }^{\circ} \mathrm{C}$ in $5 \% \mathrm{CO}_{2}$ humidified for $30 \mathrm{~min}$. Following the exposure of conditioned medium of AZM-treated RA FLSs for $24 \mathrm{~h}$, human umbilical vein endothelial cells (HUVECs; $2 \times 10^{5}$ cells $/ \mathrm{mL}$ ) were trypsinized, resuspended, and added to each chamber in sterile medium (supplemented with $5 \%$ fetal bovine serum, $100 \mathrm{U} / \mathrm{mL}$ penicillin, and $80 \mathrm{U} / \mathrm{mL}$ streptomycin) with vascular endothelial growth factor (VEGF; $50 \mathrm{ng} / \mathrm{mL}$, AF-100, Peprotech, Rocky Hill, NJ, USA), then incubated for $6 \mathrm{~h}$ at $37^{\circ} \mathrm{C}$ in $5 \% \mathrm{CO}_{2}$. After that, the capillary-like tube formation of each well in the culture plates was photographed. All experiments were done in triplicate.

\section{Quantitative real-time PCR (qRT-PCR) and Enzyme-linked immunosorbent assay (ELISA)}

Total RNA was isolated from cultured cells using TRIzol Reagent (Invitrogen) according to the manufacturer's protocol. RNA was reverse-transcribed using a ReverTra Ace qPCR RT Kit (Vazyme, China) and qRT-PCR was performed using Light-Cycler 480 (Basel Roche, Switzerland). All primers were synthesized by BGI (Beijing, China) and the sequences of all primers used in this study are listed in Online Supplementary Table 2. Relative mRNA levels are measured using the 2- $\Delta$ Cycle Threshold (2- $\Delta$ CT) method. Three independent experiments were performed, and each reaction was repeated three times.

Following treatment with AZM or ETC, cell culture supernatants were collected for the measurement of IL$1 \alpha$, IL-1 $\beta$, IL-6, IL-8, TNF- $\alpha$, MMP-1, MMP-3, CXCL9, CXCL10 and VEGF using ELISA kits (Hangzhou Multi-Science Company of China). Fresh blood was extracted from 20 -week-old mice treated with indicated drugs, and the serum was collected for measuring the release of IL-6, IL-1 $\beta$, COMP, IL-10, IL-13, RANKL and OPG by ELISA kits (Hangzhou Multi-Science Company of China).

\section{Small interfering RNA (siRNA) transfection and RNA sequencing}

Small interfering RNA (siRNA) for GRP78 and negative control was sourced from Ruibo (Guangzhou, China). siRNA targeting GRP78 or negative control siRNA was transfected into RA FLSs using transfection reagent (polyplus) according to the manufacturer's instructions. For RNA sequencing, TNF- $\alpha$ or IL- $1 \beta$ activated RA FLSs were treated with AZM for 24 hours. Then, total RNA was extracted by TRIzol method to construct a cDNA library for RNA transcriptome sequencing by LC-BIO Technologies CO., LTD (Hangzhou, China).

\section{Western blot analysis and Co-immunoprecipitation (co-IP) assay}

Western blot and Co-IP were performed as described previously (L. Wang et al., 2018). The primary antibodies for IRE1 $\alpha$ (ab37073, 1:1000), p-IRE1 $\alpha$ (ab48187, 1:1000), PERK (ab79483, 1:1000), eIf2 $\alpha$ (ab264254, 1:1000), ATF4 (ab23760, 1:1000), p38 (ab170099, 1:1000), p-p38 (ab178867, 1:1000) and p-Akt (ab38449, 1:800) were purchased from Abcam. Antibodies against ATF6 $\alpha$ (p50) (\#65880, 1:1000), p-PERK (\#3179, 1:1000) and p-eIf2 $\alpha(\# 3398,1: 1000)$ were purchased from Cell Signaling. Antibodies about CREB3L2 (14514-1-AP, 1:800), Akt (10176-2-AP, 1:2000), GRP78 (11587-1-AP, 1:2000), GFP tag (50430-2-AP, 1:1500), CHOP(15204-1-AP, 1:500), SREBP1 (14088-1-AP, 1:1000) were purchased from Proteintech. $\beta$-Actin (AF5003, 1:1000) was purchased from Beyotime. was purchased from Santa Cruz. Samples were subjected to standard SDS-PAGE, and the resulting bands were transferred onto polyvinylidene difluoride membrane for visualizing specific proteins. The ECL Plus detection system (Thermo Scientific, USA) was used for detection.

Drug Affinity Responsive Target Stability (DARTS) assay and Mass spectrometry (MS) 
Approximately $1 \times 10^{7}$ of untreated RA FLSs were lysed with M-PER Mammalian Protein Extraction Reagent (Thermo Scientific) containing protease and phosphatase inhibitors by shaking on ice for 20 minutes. 10× TNC buffer ( $1 \mathrm{M}$ Tris-HCl $5 \mathrm{ml}, 5 \mathrm{M} \mathrm{NaCl} 1 \mathrm{ml}, 1 \mathrm{M} \mathrm{CaCl}_{2} 1 \mathrm{ml}$, double distilled $\mathrm{H}_{2} \mathrm{O} 3 \mathrm{ml}$, $\mathrm{PH}$ 7.4) was added into the cell lysate $(500 \mu \mathrm{g}$ total protein, $5 \mathrm{mg} / \mathrm{mL})$ to reach the indicated concentrations and gently mixed. AZM and the protein lysate were incubated for 1 hour at room temperature to allow the target protein and ligand to sufficiently bind, and then digested by pronase (Roche, dissolved in $1 \times$ TNC buffer) at a 1:1000 ratio (wt/wt) for precisely $15 \mathrm{~min}$ at room temperature. After drug incubation and protease digestion of protein cleavage products, $5 \times$ protein loading buffer was added and boiled for 5 minutes. Then SDS-PAGE was performed, and the bands were visualized by Coomassie blue staining. Finally, the gel of bands with obvious changes were identified by mass spectrometry (BGI, China) (Online Supplementary Table 3).

\section{Cellular thermal shift assay (CETSA)}

CETSA can directly detect the binding affinity of drugs and target proteins in cells or tissues. Briefly, cells were seeded in $10 \mathrm{~cm}$ plates and grown to $80 \%-90 \%$ confluence. The cells were treated with different concentrations of AZM or dimethyl sulfoxide for 1 hour. After treatment, the cells were trypsinized, washed twice with PBS, and then resuspended in PBS solution containing protease inhibitors, and heated at $42{ }^{\circ} \mathrm{C}$, $46{ }^{\circ} \mathrm{C}, 49{ }^{\circ} \mathrm{C}, 52{ }^{\circ} \mathrm{C}, 54{ }^{\circ} \mathrm{C}$, and $57^{\circ} \mathrm{C}$ for 3 minutes. Then, following freezing and thawing, the samples were centrifuged at $12,000 \mathrm{rpm}$ for $20 \mathrm{~min}$ at $4{ }^{\circ} \mathrm{C}$ and analyzed by western blot.

\section{Plasmids transfection}

The DNA fragments corresponding to the full-length GRP78 were amplified by polymerase chain reaction (PCR) from Marathon-Ready HeLa cDNA. Human GRP78 cDNA encompassing full length, deletions lacking peptide-binding region or ATPase domain were amplified by reverse transcription PCR and cloned into pCR8/GW/TOPO (K280020, Invitrogen) as described before (Goehring et al., 2014). The amino acid residue, Asp180, locates in the ATPase domain of GRP78 and is essential for catalysis. The D180A mutants of GRP78 were prepared with the QuikChange site-directed mutagenesis kit (200523, Agilent, CO, USA), as previously described (Sawano \& Miyawaki, 2000). All deletion and point mutations were made by PCR and confirmed by DNA sequencing.

\section{GRP78 protein ATPase activity assay}

RA FLSs were seeded in $10 \mathrm{~cm}$ cell culture dishes and transfected with GRP78 recombinant plasmid. After 48 hours of transfection, cells were scraped to extract protein. ATPase activity is determined using KinaseLumi Chemiluminescence Kinase Activity Detection Kit (Beyotime Biotechnology). Specifically, ATP with different concentrations was diluted by kinase reaction buffer and $50 \mu \mathrm{l}$ Kinase-Lumi Chemiluminescence Kinase Detection Reagent was then added. Following reaction at room temperature (about $25{ }^{\circ} \mathrm{C}$ ) for 10 minutes, chemiluminescence detection was performed with a multifunctional microplate reader to draw an ATP standard curve. After measuring the value of GRP78 protein substrate, the enzyme activity was calculated according to the standard curve.

\section{CRISPR/Cas9-mediated knockout of GRP78}

For lentiviral experiments, three sgRNA sequences targeting GRP78 were selected based on enrichment scores from the whole genome CRISPR screens and cloned into the lentiCRISPR v2 (Addgene plasmid no. 52961) according to the protocol provided by the Zhang Lab (Zuo et al., 2017). A complete list of CRISPR sequences can be found in Online Supplementary Table 4. Lentivirus was generated by co-transfecting HEK293T cells with lentiCRISPR v2, envelop plasmid pMD2.G (Addgene plasmid no. 12259), and packaging plasmid psPAX2 using Lipofectamine 2000 (Invitrogen, Carlsbad, CA, USA), Viral supernatants were collected 48 hours following transfection and used to infect HEK293T cells. Puromycin selection ( $3 \mu \mathrm{M}, \mathrm{HY}-15695, \mathrm{MCE})$ was performed for 3 to 4 days establish the stable cell lines.

\section{Collagen-induced arthritis (CIA) model}


Nine-week-old male DBA/1 mice purchased from Vital River Laboratory Animal Technology (Beijing, China) were raised in accordance with institutional guidelines and with the approval of the Institutional Animal Care and Use Committee of Shandong First Medical University \& Shandong Academy of Medical Sciences. CIA models were constructed and established as described before (Song, Feng, Zhao, Lu, \& Han, 2019). Mice immunized with CII were randomly divided into 4 groups ( $\mathrm{n}=6$ mice for each group and time point) and administered AZM or Vehicle at the indicated doses twice a day after the initial immunization. In the therapeutic treatment study, when arthritis score reached 6 , mice were randomly enrolled into groups. AZM, ETC or Vehicle (6 mice per group) were administered i.p. to mice, twice a day from the day of enrollment. Progression of arthritis was monitored continuously after the first immunization by assessing arthritis score (the sum of scores on a 0-4 scale for all 4 limbs, based on the swelling of the limbs, for a total possible score of 16 for each mouse), using methods as described before. The arthritis score for each mouse was defined as the sum of the scores of all four paws. In each category, joints were independently evaluated in a blinded manner. In addition, the thickness of the arthritic hind paws was measured daily with microcalipers.

\section{Hematoxylin-Eosin (H\&E) staining and Histopathological examination}

After decalcification of mice joints in 10\% EDTA, together with human synovial tissues, they were embedded in paraffin, and stained with $\mathrm{H} \& \mathrm{E}$ staining for light microscopy. Histopathological quantification was made by investigators in a blinded manner from images of three fields representing the distal interphalangeal joint, the proximal interphalangeal joint, and joints in the carpal region and averaged over all joints in one limb of each animal. Inflammation was scored 0 to 5 according to the following criteria: 0 , normal; 1 , minimal inflammatory infiltration; 2, mild infiltration; 3, moderate infiltration with moderate edema; 4, marked infiltration with marked edema; and 5, severe infiltration with edema. Hyperplasia was defined as synovial tissue intimately invading bone and/or cartilage and was scored 0 to 3 as follows: 0 , none; 1 , minimal; 2, moderate; 3 , severe. Bone degradation was scored using the following 0 to 3 scale: 0 , no bone erosion; 1 , mild surface erosion; 2, moderate surface erosion; and 3, strong surface erosion. Sections were also stained by Safranin O/ Fast green kit (Solarbio) for detection of cartilage damage (0: no changes; 1: erosion in part of the cartilage surface; 2: erosion of the cartilage surface and cartilage destruction; 3: cartilage erosion and destruction, combined with cartilage covered by connective tissue; 4: Cartilage and cartilage covered by connective tissue are corroded and damaged severely).

\section{Micro-Computed Tomography (Micro-CT)}

Micro-CT (QuantumGX, PerkinElmer, United States) was conducted on the hind paw to scan the microstructure of the joint and parameters were analyzed precisely at the same region of distal tibia. Specimen scanner settings were designed as follows: exposure time $14 \mathrm{~min}$ at $90 \mathrm{KV}$ and $88 \mathrm{~mA}$ with a resolution of $2 \mu \mathrm{m}$ and field-of-view $12.8 \mathrm{~mm} \times 12.8 \mathrm{~mm}$. The parameters of trabecular bone, including bone volume to bone volume fraction (BV/TV, \%), trabecular thickness (Tb.Th, $\mu \mathrm{m})$, trabecular bone mineral density (tb.BMD, g/cm3), trabecular number (Tb.N, mm/1), trabecular separation (Tb.Sp, mm) were exported for reconstruction using the manufacturer's software, Caliper Analyze.

\section{Statistical analysis}

The arthritis scores and paw swelling were analyzed with the Mann-Whitney U test if the requirement of Gaussian distribution and homogeneity of variance weren't met. Differences in the levels of inflammatory factors and micro-CT parameters between the groups were analyzed with one-way ANOVA for Homogeneity test of variance and we fulfilled nonparametric Mann Whitney $\mathrm{U}$ test for the comparison between two groups due to the heterogeneity of variances. Numbers of peripheral blood cells, GRP78 mRNA levels and histological score were confirmed to Gaussian distribution and homogeneity of variances and were compared with Student's unpaired t-test. Data were expressed as mean \pm standard errors (SD) of the means. SPSS software (version 22.0; SPSS Inc., Chicgo, IL, USA) were used to accomplished all statistical analysis. Two-sided $P$ values of less than 0.05 were considered as statistical significance.

\section{RESULTS}




\section{AZM treatment ameliorates the arthritis phenotype of RA FLSs}

We firstly analyzed the effects of AZM on the inflammatory phenotype of RA FLSs. and AZM treatment dose-dependently reduced the levels of IL-6 (Fig. 1A), IL-8 (Fig. 1B), MMP-1 (Fig. 1C) and MMP-3 (Fig. 1D) irrespective of TNF- $\alpha$ or IL-1 $\beta$ stimulation. Furthermore, AZM treatment reduced the migration (Fig. 1E) and invasion (Fig. 1F) of RA FLSs. Similarly, there was a strong reduction in CXCL9 (Fig. 1G) and CXCL10 levels (Fig. 1H) in RA FLSs and migrating leucocytes (Fig. 1I) cultured with AZM. In addition, the production of VEGF (Fig. 1J), as well as the pro-angiogenensis ability (Fig. 1K), also decreased when RA FLSs were exposed to AZM. Thus, these data establish the importance of AZM in suppressing the inflammatory phenotype of RA FLSs.

TNF antagonists are the most widely used biological disease-modifying anti-rheumatic drug in RA (Van Schouwenburg, Rispens, \& Wolbink, 2013). However, following consideration of the uncertainty of its therapeutic effects and its high price, it is worthy to explore novel treatment strategy. Importantly, AZM reduced the inflammatory factors above as effectively as Etanercept. However, no additive effects were observed when AZM and Etanercept were introduced simultaneously (online Supplementary Fig. 1). Besides, consistent with previous study demonstrating that azithromycin alters macrophage phenotype, in this study, we further confirmed that AZM treatment decreased the production of IL-6, IL- 8 , TNF- $\alpha$, IL- $1 \alpha$ and IL- $1 \beta$ in PBMC from RA patients (online Supplementary Fig. 2). Therefore, more details would be needed to further support its potential as novel treatment drug for RA.

\section{Effects of therapeutic treatment with AZM in arthritis models}

We next determined the impact of AZM in mouse model of rheumatoid arthritis: CIA. Compared with solvent-treated ones, AZM-treated mice showed dose-dependent amelioration with a marked decrease in arthritis score (Fig. 2A), thickness (Fig. 2B) and paw swelling (Fig. 2C). Histological analysis of affected joints from AZM-treated mice revealed reduced evidence of synovial hyperplasia, cartilage degradation and bone destruction (Fig. 2D). The score data showed that the degree of inflammation, synovial hyperplasia, cartilage degradation and bone destruction were reduced in AZM treated mice (Fig. 2E). In addition, AZMtreated CIA mice displayed reduced levels of bone erosion and destruction (Fig. 2F), as evidenced by the increase in BV/TV, Tb. BMD, Tb. Th and Tb. $\mathrm{N}$ but decrease in Tb. Sp in the distal tibia (Fig. 2G). Mice treated with AZM also had significantly decreased serum levels of proinflammatory cytokines interleukin (IL)-1 $\beta$ and IL-6, and COMP, and elevated levels of anti-inflammatory cytokines IL-10 and IL-13, when compared with control mice (online Supplementary Fig. 3). Additionally, AZM treatment decreased the RANKL level and increased the OPG/RANKL ratio (online Supplementary Fig. 3).

\section{AZM is identified as an antagonist of UPR signaling and activity}

The transcriptome sequencing (RNA-seq) analysis was applied to identify the differentially expressed genes (DEGs) in TNF- $\alpha$ or IL-1 $\beta$-activated RA FLSs with AZM treatment, and 198 genes and 217 genes were significantly differentially expressed, respectively (Fig. 3A). Then, enrichment analysis of GO classification and KEGG pathway was conducted. Among the biological processes, 68 genes and 52 genes of the DEGs were enriched in the biosynthesis and metabolism of lipid, cholesterol and steroid, cell proliferation, protein exit from ER, SREBP-SCAP complex retention in ER, redox process and regulation of transcription (Fig. 3B). KEGG analysis showed that 66 genes and 64 genes of the DEGs were mainly involved in Signal transduction, Immune system, Cholesterol and Glycerolipid metabolism, PPAR, AMPK, TNF and PI3K-Akt signaling pathway (Fig. 3C). qRT-PCR was used to confirm the results of RNA-seq analysis that the expression trends of genes involved in cholesterol biosynthetic process (CYP51A1, ACAT2, PCSK9),lipid metabolic process (MSMO1, IDI1, SCD, PNPLA3, LIPG, SC5D, HSD17B7, FADS2) were increased following AZM treatment (online Supplementary Fig. 4). Several other genes, involved in steroid biosynthetic process (LDLR and ABCA1) and apoptosis (HRK and TUBA1A), were also found and validated to be increased (online Supplementary Fig. 4).

Intriguingly, in the cellular component field of TNF- $\alpha$ or IL-1 $\beta$-activated RA FLSs with AZM treatment, 32 and 28 DEGs were distributed to ER (Fig. 3B). ER is the site of cholesterol- or lipid-induced cytotoxicity 
in various cell types (Yeo et al., 2014). Accumulation of free cholesterol (FC) and lipid in the ER leads to activation of the unfolded protein response (UPR) and C/EBP homologous protein (CHOP)-induced apoptosis (Erdi, Sozen, Kartal, \& Ozer, 2017). Then, we tested the effect of AZM on the UPR effector, CHOP. As shown in Fig. 3D, CHOP was induced markedly $12 \mathrm{~h}$ post AZM exposure. Furthermore, the activity of UPR components enhanced as evidenced by the increased phosphorylation of PERK and elF $2 \alpha$, and the up-regulated expression of IRE1 $\alpha$ and, ATF4 (Fig. 3E). RA FLSs are characterized by apoptotic resistance against ER stress (Rahmati et al., 2018). In this study, the expression of p-p38, CREB3L2 and p-Akt which represents pro-apoptotic markers was obviously upregulated after AZM treatment (Fig. 3F). Furthermore, in the presence of ER stress inducer, more proportion of apoptosis were found when RA FLSs were treated AZM as compared to its control (Fig. 3G). Taken together, AZM treatment may disturb the biosynthetic process of cholesterol, lipid and steroid, activate UPR and subsequently induce apoptosis of RA FLSs.

\section{GRP78 is a novel target of AZM}

To clarify the molecular basis underlying how AZM imposed its effects on RA FLSs, we aimed to isolate protein binding partners of AZM. By implementing DARTS assay and mass spectrometry, glucose-regulated protein 78 (GRP78) was identified as potential candidates (Fig. 4A). Then, we performed Western blot of DARTS samples with which a series of protease to cell lysate ratios were implemented, and found that AZM protected GRP78 (Fig. 4B). Subsequently, we conducted CETSA, which allows for quantification of the change in thermal denaturation temperature of a target protein under different conditions, including those of varying temperature and concentrations of drug (X. Wang et al., 2013). AZM increased the resistance of GRP78 in RA FLSs to denaturation caused by heating from $52{ }^{\circ} \mathrm{C}$ to $58{ }^{\circ} \mathrm{C}$ compared with DMSO (Fig. 4C). Furthermore, AZM increased stability of GRP78 in a dose-dependent manner (Fig. 4D).

In order to identify the domain by which AZM targets to GRP78, recombinant molecules were produced and the DARTS assay revealed that AZM retained protective effects on mutant GRP78 lacking peptide-binding region while showed no protective effects on mutant lacking ATPase domain (Fig. 4E). Then, molecular docking of AZM towards GRP78 was carried out using DOCK6 (Hu et al., 2012) and their potential interacting sites were presented in Fig. 4F. AZM showed a salt-bridge interaction with residue Asp178 of the GRP78 protein. It also formed H-bond interactions with residue Arg181, Ile199, Val241 and Gln401. Importantly, DARTS assay clearly demonstrated that AZM lost its protective effect upon substituting Asp178 of GRP78 into Ala (Fig. 4G), demonstrating that Asp178 is the critical amino acid required for AZM targeting to GRP78.

Since the ATPase domain of GRP78 is required for its activity upon binding with ATP (Galligan et al., 2014), AZM might competitively bind GRP78 with ATP. As expected, the enzyme activity of GRP78 was competitively inhibited by AZM in the presence of ATP (Fig. 4H). HA15 is able to inhibit the BiP activity via directly suppressing its ATPase activity (Cerezo et al., 2016). Importantly, AZM could inhibit GRP78 activity as efficiently as HA15 (Fig. 4I). Thus, GRP78 serves as a novel substrate for AZM in RA FLSs.

\section{AZM activates UPR activity through facilitating the dissociation of GRP78 from its interactors}

The activation of ER stress occurred upon the dissociation of GRP78 from the luminal part of the ER integral membrane proteins: PERK, IRE1 $\alpha$ and ATF6 $\alpha$ (Yoo et al., 2013). Co-immunoprecipitation experiments showed that incubation of RA FLSs lysates with AZM decreased the GRP78 level in the PERK, IRE1 $\alpha$, and ATF6 $\alpha$ immunoprecipitated fractions (Fig. 5A), thus indicating that AZM induced a dissociation of the GRP78, PERK, IRE1 $\alpha$ and ATF6 $\alpha$ complex and representing the initial event necessary to induce the observed ER stress.

Furthermore, SREBP-SCAP complex is like other key integral membrane proteins of the UPR associated with GRP78, and, the export of the complex requires its dissociation from GRP78 (Jin et al., 2000). GRP78 dissociates from SREBP allows its cleavage and nuclear translocation for its regulatory function (Katanasaka et al., 2010). Furthermore, attenuation of ER stress by overexpression of GRP78 blocked SREBP activation and decreased the expression of genes responsible for cholesterol and fatty acid biosynthesis (Kammoun et 
al., 2009). Here, an enhanced expression of m-SREBP-1c by AZM was seen in nuclear extract of RA FLSs, but not in cytoplasmic protein (Fig. 5B). Furthermore, the enhancement of m-SREBP-1c (mature SREBP1c) nuclear translocation resulting from AZM treatment could be attenuated by overexpression of GRP78 in RA FLSs (Fig. 5B). These data supported that AZM inhibits the activity of GRP78, thereby provoking ER stress and activating SREBP-mediated cholesterol and lipid metabolism.

\section{GRP78 is required for the anti-arthritis effects of AZM}

We deleted GRP78 gene using the CRISPR-Cas9 technique and this technique produced nearly complete deletion of GRP78 (Fig. 6A). Although GRP78 deficiency resulted in the increase of PERK and elF2 $\alpha$ phosphorylation, IRE1 $\alpha$, ATF4 and CHOP expression, as well as SREBP nuclear translocation in the absence of AZM, no further changes were observed when GRP78-/- were challenged with AZM (Fig. 6B). In addition, re-establishing expression of GRP78 by transfecting GRP78 knockout cells with a GRP78 expression plasmid reinstated AZM's UPR and SREBP activation (Fig. 6B). However, transfection of GRP78 knockout cells with an expression plasmid encoding a GRP78 point mutant Asp-178-Ala (GRP78 D178A), which inactivates GRP78 enzymatic activity and fails to hydrolyze ATP, could not rescue AZM's activity (Fig. 6B). Taken together, these findings indicate the dependence of AZM's UPR activation on GRP78 and GRP78-mediated ATPase activity.

To further ascertain that the anti-arthritis effect of AZM is mediated by GRP78, GRP78 was inhibited by siRNA in RA FLSs (Fig. 6C). The downregulation of GRP78 reduced the production of inflammatory factors (Fig. 6D) and genes involved in cholesterol and lipid metabolism process (online Supplementary Fig. 4), but induced apoptosis (Fig. 6E), which was the same as the effect of AZM. However, coculture with AZM had no synergistic effect on inflammatory factors, genes of cholesterol and lipid metabolism, and cell apoptosis with GRP78 knockdown (Fig. 6D, 6E, and online Supplementary Fig. 4). Taken together, our results showed that AZM directly bound to GRP78 and exerted anti-inflammatory effects in RA.

\section{DISCUSSION}

Recent years, the use of antibiotics (primarily the tetracycline analogues) to treat RA (van der Veen, van der Heide, Kruize, \& Bijlsma, 1994) has seen a resurgence of interest as they interfere with the production of prostaglandins and leukotrienes, scavenges oxygen radicals, as well as the expression of nitrous oxide synthase (Porstmann et al., 2005), and enhances natural inhibitors of matrix metalloproteinases (Pastorino \& Shulga, 2008). Consistent with previous study suggesting the potential efficacy of AZM in arthritic rats (Carevi \& Djoki, 1988), in this study, we found that AZM effectively suppressed principal indices of arthritis in the CIA model, as evidenced by the decrease of joints swelling, articular cartilage and bone destruction, deformity and synovitis. These data provide further evidence to support the therapeutic potential of AZM in RA. Importantly, AZM could ameliorate the inflammatory phenotype in CIA models as effectively as TNF- $\alpha$ blocker, thus suggesting an alternative intervention to overcome the drug insensitivity and high price of TNF- $\alpha$ inhibitors in RA treatment.

Together with our previous studies, chronic ER stress could be an underlying cause of synovial cell proliferation and production of pro-inflammatory cytokines (Geng et al., 2020). Besides, RA FLSs are resistant to apoptosis induced by ER stress (Kabala et al., 2017). Therefore, the components of ER stress have become an interesting target for RA therapy. The connection between ER stress and RA began to evolve after the contribution of GRP78 to the pathogenesis of RA(Yoo et al., 2012). During ER stress, the increase of GRP78 expression could amplify the inflammatory cascade by preventing RA FLSs from apoptotic death and escalating pannus formation. GRP78 knockdown markedly reduces vascular endothelial growth factor (VEGF)-induced angiogenesis, inhibits cytokine-mediated proliferation and invasion, and induces apoptosis of FLSs in mouse models of RA, thus presenting as a cross-point for multiple pathological phenotypes in RA (Song et al., 2019). Indeed, GRP78/BiP inhibition served as an effective approach to suppress the pathogenic features of RA, particularly for those patients with treatment-resistant RA. Additionally, as recombinant human GRP78/BiP is currently in Phase I/II clinical trials in patients with RA, and the outcomes are promising. Therefore, both the inhibition and expression of GRP78/BiP can be of therapeutic benefit 
in RA, depending on the clinical scenario. In this study, our findings that the commonly used antibiotics drug AZM targets GRP78 and inhibits its ATPase activity, further supporting the potential of AZM in RA treatment.

Cholesterol and lipids are essential for the proper biological function of cellular membranes, whereas excess cholesterol and lipids can be toxic and even lead to apoptosis. Previous study showed that SREBP activation leads to the induction of key enzymes of the cholesterol and fatty acid biosynthesis pathways, and thus membrane homeostasis (Porstmann et al., 2005). However, in response to ER stress, SREBPs overexpression increased the expression of cholesterolgenic and lipogenic gene, resulting in cholesterol and lipid accumulation and provoking consequent apoptosis. Three potential mechanisms by which ER stress induces SREBP activation: caspase-induced SREBP cleavage (Colgan, 2007), eIF2 $\alpha$-phosphorylation- dependent downregulation of INSIG (Birkenfeld et al., 2011) and GRP78 dissociation from the SCAP-SREBP complex (Su et al., 2019). Previous studies showed that in vitrooverexpression of GRP78 could decrease markers of ER stress, attenuate ER-stress-induced SREBP activation and lipid accumulation (Lhoták et al., 2012). Here, we examined the changes of molecular events occurred in RA FLSs following AZM treatment and the data showed that the significantly dysregulated genes were involved mainly in cholesterol and lipid biosynthetic process. Consistently, detailed analysis showed that AZM treatment promoted the dissociation of SREBP away from GRP78 and increased the expression of SREBP targeted genes. In essence, the evidence presented here marks UPR activation as an additional effector program by which AZM exerts its anti-arthritis potential, and argues for a GRP78-mediated mechanism as the underlying mode of AZM's molecular basis for RA treatment.

Initial characterizations of AZM have demonstrated functional roles in controlling acute and chronic inflammatory responses, both in vitro and in vivo (Banjanac et al., 2012). In these studies, anti-inflammatory activity was observed on monocytes, lymphocytes, microphage, epithelial cells and fibroblasts, all cell populations which contribute to pathology in RA and other forms of inflammatory arthritis (Kvien et al., 2004). Therefore, the therapeutic effects of AZM might be caused by immunomodulatory activities towards different cells types, even though the direct effect of AZM on these cells in CIA mice or in RA in vivo must be further explored.

In summary, the specific AZM significantly decreased the severity of CIA, reduced the production of proinflammatory cytokines, and prevented in vivo bone destruction in CIA models. Thus, our study provides AZM as an alternative intervention for RA treatment that may overcome the drug insensitivity and high price of TNF- $\alpha$ inhibitors, and further support the components of UPR as promising treatment targets for RA.

\section{REFERENCES}

Ai, R., Laragione, T., Hammaker, D., Boyle, D. L., Wildberg, A., Maeshima, K., . . . Firestein, G. S. (2018). Comprehensive epigenetic landscape of rheumatoid arthritis fibroblast-like synoviocytes. Nat Commun, 9 (1), 1921. doi:10.1038/s41467-018-04310-9

Banjanac, M., Munić Kos, V., Nujić, K., Vrančić, M., Belamarić, D., Crnković, S., . . . Eraković Haber, V. (2012). Anti-inflammatory mechanism of action of azithromycin in LPS-stimulated J774A.1 cells.Pharmacological research, 66 (4), 357-362. doi:10.1016/j.phrs.2012.06.011

Bartok, B., \& Firestein, G. S. (2010). Fibroblast-like synoviocytes: key effector cells in rheumatoid arthritis. Immunological Reviews, 233 (1), 233-255.

Birkenfeld, A., Lee, H., Majumdar, S., Jurczak, M., Camporez, J., Jornayvaz, F., . . . Shulman, G. (2011). Influence of the hepatic eukaryotic initiation factor 2alpha (eIF2alpha) endoplasmic reticulum (ER) stress response pathway on insulin-mediated ER stress and hepatic and peripheral glucose metabolism. The Journal of biological chemistry, 286 (42), 36163-36170. doi:10.1074/jbc.M111.228817

Carevi, O., \& Djoki, S. (1988). Comparative studies on the effects of erythromycin A and azithromycin upon extracellular release of lysosomal enzymes in inflammatory processes. Agents and Actions, 25 (1-2), 124-131. 
Cerezo, M., Lehraiki, A., Millet, A., Rouaud, F., Plaisant, M., Jaune, E., . . . Rocchi, S. (2016). Compounds Triggering ER Stress Exert Anti-Melanoma Effects and Overcome BRAF Inhibitor Resistance.Cancer Cell, 30 (1), 183. doi:10.1016/j.ccell.2016.06.007

Colgan, S. (2007). Endoplasmic reticulum stress causes the activation of sterol regulatory element binding protein-2. Int J Biochem Cell Biol, 39 .

Cory, T., Birket, S., Murphy, B., Mattingly, C., Breslow-Deckman, J., \& Feola, D. (2013). Azithromycin increases in vitro fibronectin production through interactions between macrophages and fibroblasts stimulated with Pseudomonas aeruginosa. The Journal of antimicrobial chemotherapy, 68 (4), 840-851. doi:10.1093/jac/dks476

Erdi, Sozen, Kartal, N., \& Ozer. (2017). Impact of high cholesterol and endoplasmic reticulum stress on metabolic diseases: An updated mini-review. Redox biology .

Galligan, J. J., Fritz, K. S., Backos, D. S., Shearn, C. T., Smathers, R. L., Jiang, H., . . . Petersen, D. R. (2014). Oxidative stress-mediated aldehyde adduction of GRP78 in a mouse model of alcoholic liver disease: functional independence of ATPase activity and chaperone function. Free Radical Biology 83 Medicine, 73 , 411-420.

Geng, M., Xu, K., Meng, L., Xu, J., Jiang, C., Guo, Y., . . . Lu, S. (2020). Up-regulated DERL3 in fibroblastlike synoviocytes exacerbates inflammation of rheumatoid arthritis. Clinical immunology (Orlando, Fla.), 220 , 108579. doi:10.1016/j.clim.2020.108579

Goehring, A., Lee, C. H., Wang, K. H., Michel, J. C., Claxton, D. P., Baconguis, I., . . . Gouaux, E. (2014). Screening and large-scale expression of membrane proteins in mammalian cells for structural studies. Nature Protocols, 9 (11), 2574.

Haydar, D., Cory, T. J., Birket, S. E., Murphy, B. S., Pennypacker, K. R., Sinai, A. P., \& Feola, D. J. (2019). Azithromycin Polarizes Macrophages to an M2 Phenotype via Inhibition of the STAT1 and NF-xB Signaling Pathways. Journal of immunology (Baltimore, Md. : 1950), 203 (4), 1021-1030. doi:10.4049/jimmunol.1801228

Hetz, C., \& Saxena, S. (2017). ER stress and the unfolded protein response in neurodegeneration. Nature Reviews Neurology, 13 (8).

Hu, W. J., Yan, L., Park, D., Jeong, H. O., Chung, H. Y., Yang, J. M., . . . Qian, G. Y. (2012). Kinetic, structural and molecular docking studies on the inhibition of tyrosinase induced by arabinose.International journal of biological macromolecules, 50 (3), 694-700.

Jin, Ye, and, Robert, Rawson, and, . . . and. (2000). ER Stress Induces Cleavage of Membrane-Bound ATF6 by the Same Proteases that Process SREBPs. Molecular Cell .

Kabala, P., Angiolilli, C., Yeremenko, N., Grabiec, A., Giovannone, B., Pots, D., . . . Reedquist, K. (2017). Endoplasmic reticulum stress cooperates with Toll-like receptor ligation in driving activation of rheumatoid arthritis fibroblast-like synoviocytes. Arthritis Research \& Therapy, 19 (1), 207. doi:10.1186/s13075-017$1386-\mathrm{x}$

Kammoun, H., Chabanon, H., Hainault, I., Luquet, S., Magnan, C., Koike, T., . . . Foufelle, F. (2009). GRP78 expression inhibits insulin and ER stress-induced SREBP-1c activation and reduces hepatic steatosis in mice. The Journal of clinical investigation, 119 (5), 1201-1215. doi:10.1172/jci37007

Katanasaka, Y., Ishii, T., Asai, T., Naitou, H., Maeda, N., Koizumi, F., . . . Oku, N. (2010). Cancer antineovascular therapy with liposome drug delivery systems targeted to BiP/GRP78. International Journal of Cancer Journal International Du Cancer, 127 (11), 2685-2698.

Kvien, T., Gaston, J., Bardin, T., Butrimiene, I., Dijkmans, B., Leirisalo-Repo, M., . . . Vischer, T. (2004). Three month treatment of reactive arthritis with azithromycin: a EULAR double blind, placebo controlled 
study. Annals of the Rheumatic Diseases, 63 (9), 1113-1119. doi:10.1136/ard.2003.010710

Lhoták, S., Sood, S., Brimble, E., Carlisle, R., Colgan, S., Mazzetti, A., . . Austin, R. (2012). ER stress contributes to renal proximal tubule injury by increasing SREBP-2-mediated lipid accumulation and apoptotic cell death. American journal of physiology. Renal physiology, 303 (2), F266-278. doi:10.1152/ajprenal.00482.2011

Liu, Y., Pu, Y., Li, D., Zhou, L., \& Wan, L. (2017). Azithromycin ameliorates airway remodeling via inhibiting airway epithelium apoptosis. Life sciences, 170 , 1-8. doi:10.1016/j.lfs.2016.11.024

Lu, Q., Wang, J., Zhang, X., Tian, R., Qiao, L., Ge, L., . . Wang, L. (2020). TXNDC5 protects synovial fibroblasts of rheumatoid arthritis from the detrimental effects of endoplasmic reticulum stress.Intractable $\& 3$ rare diseases research, 9 (1), 23-29. doi:10.5582/irdr.2019.01139

Mierke, C. (2019). The matrix environmental and cell mechanical properties regulate cell migration and contribute to the invasive phenotype of cancer cells. Reports on progress in physics. Physical Society (Great Britain), 82 (6), 064602. doi:10.1088/1361-6633/ab1628

Nujić, K., Banjanac, M., Munić, V., Polančec, D., \& Eraković Haber, V. (2012). Impairment of lysosomal functions by azithromycin and chloroquine contributes to anti-inflammatory phenotype. Cellular immunology, 279 (1), 78-86. doi:10.1016/j.cellimm.2012.09.007

O'Neil, L. J., \& Kaplan, M. J. (2019). Neutrophils in Rheumatoid Arthritis: Breaking Immune Tolerance and Fueling Disease. Trends Mol Med, 25 (3), 215-227. doi:10.1016/j.molmed.2018.12.008

Ozsvari, B., Nuttall, J., Sotgia, F., \& Lisanti, M. (2018). Azithromycin and Roxithromycin define a new family of "senolytic" drugs that target senescent human fibroblasts. Aging, 10 (11), 3294-3307. doi:10.18632/aging.101633

Parnham, M. J., Haber, V. E., Giamarellos-Bourboulis, E. J., Perletti, G., \& Vos, R. (2014). Azithromycin: mechanisms of action and their relevance for clinical applications. Pharmacology 83 Therapeutics, 143 (2), 225-245.

Pastorino, J. G., \& Shulga, N. (2008). Tumor necrosis factor-alpha can provoke cleavage and activation of sterol regulatory element-binding protein in ethanol-exposed cells via a caspase-dependent pathway that is cholesterol insensitive. The Journal of biological chemistry, 283 (37), 25638-25649. doi:10.1074/jbc.M800237200

Porstmann, T., Griffiths, B., Chung, Y., Delpuech, O., Griffiths, J., Downward, J., \& Schulze, A. (2005). $\mathrm{PKB} /$ Akt induces transcription of enzymes involved in cholesterol and fatty acid biosynthesis via activation of SREBP. Oncogene, 24 (43), 6465-6481. doi:10.1038/sj.onc.1208802

Rahmati, M., Moosavi, M., \& McDermott, M. (2018). ER Stress: A Therapeutic Target in Rheumatoid Arthritis? Trends in Pharmacological Sciences, 39 (7), 610-623. doi:10.1016/j.tips.2018.03.010

Reverendo, M., Mendes, A., Argüello, R., Gatti, E., \& Pierre, P. (2019). At the crossway of ER-stress and proinflammatory responses. The FEBS journal, 286 (2), 297-310. doi:10.1111/febs.14391

Sawano, A., \& Miyawaki, A. (2000). Directed evolution of green fluorescent protein by a new versatile PCR strategy for site-directed and semi-random mutagenesis. Nucleic Acids Research, 28 (16), E78. doi:10.1093/nar/28.16.e78

Song, G., Feng, T., Zhao, R., Lu, Q., \& Han, J. (2019). CD109 regulates the inflammatory response and is required for the pathogenesis of rheumatoid arthritis. Annals of the Rheumatic Diseases, 78 (12), annrheumdis2019-215473.

Spagnolo, P., Fabbri, L. M., \& Bush, A. (2013). Long-term macrolide treatment for chronic respiratory disease. European Respiratory Journal, 42 (1), 239-251. 
Su, L., Zhou, L., Chen, F.-J., Wang, H., Qian, H., Sheng, Y., . . . Li, P. (2019). Cideb controls sterolregulated ER export of SREBP/SCAP by promoting cargo loading at ER exit sites. The EMBO journal, 38 (8), e100156. doi:10.15252/embj.2018100156

van der Veen, M. J., van der Heide, A., Kruize, A. A., \& Bijlsma, J. W. (1994). Infection rate and use of antibiotics in patients with rheumatoid arthritis treated with methotrexate. Annals of the Rheumatic Diseases, 53 (4), 224-228. doi:10.1136/ard.53.4.224

Van Schouwenburg, P. A., Rispens, T., \& Wolbink, G. J. (2013). Immunogenicity of anti-TNF biologic therapies for rheumatoid arthritis.Nature Reviews Rheumatology, 9 (3), 164-172.

Vanaudenaerde, B. M., Wuyts, W. A., Geudens, N., Dupont, L. J., \& Verleden, G. M. (2010). Macrolides inhibit IL17-induced IL8 and 8-isoprostane release from human airway smooth muscle cells.American Journal of Transplantation, 7 (1), 76-82.

Wang, L., Dong, H., Song, G., Zhang, R., Pan, J., \& Han, J. (2018). TXNDC5 synergizes with HSC70 to exacerbate the inflammatory phenotype of synovial fibroblasts in rheumatoid arthritis through NF- $x \mathrm{~B}$ signaling. Cellular \& molecular immunology, 15 (7), 685-696. doi:10.1038/cmi.2017.20

Wang, L., Song, G., Zheng, Y., Wang, D., Dong, H., Pan, J., \& Chang, X. (2016). miR-573 is a negative regulator in the pathogenesis of rheumatoid arthritis. Cellular \& molecular immunology, 13 (6), 839-849. doi:10.1038/cmi.2015.63

Wang, X., Dou, Z., Yuan, Y., Man, S., Wolfs, K., Adams, E., \& Van Schepdael, A. (2013). On-line screening of matrix metalloproteinase inhibitors by capillary electrophoresis coupled to ESI mass spectrometry. Journal of Chromatography B Analytical Technologies in the Biomedical $\mathcal{E}$ Life ences, 930 , 48-53.

Weijun, W., Ming, Q., Wanwan, J., Zheng, H., Zhongzheng, L., Di, Y., . . . Jianchun, M. (2018). Cystathionine- $\gamma$-lyase ameliorates the histone demethylase JMJD3-mediated autoimmune response in rheumatoid arthritis. Cellular 83 molecular immunology, 16 .

Xu, Yang, Berezowska, Gao, \& Peng. (2019). Endoplasmic Reticulum Stress Signaling as a Therapeutic Target in Malignant Pleural Mesothelioma.Cancers, 11 (10), 1502-.

Yeo, L., Lom, H., Juarez, M., Buckley, C., Filer, A., Raza, K., \& Scheel-Toellner, D. (2014). A1.53 A novel pro-inflammatory B cell population in the rheumatoid synovium can be identified by expression of FCRL4. Annals of the Rheumatic Diseases, 73 (Suppl 1), A22-.

Yoo, W. H., Sung, M. S., Lee, E. G., Lee, C. H., Lee, M. S., Cheon, Y. H., \& Lee, S. I. (2013). AB0138Altered er stress-induced autophagic reactions are associated with increased apoptosis of tymphocytes in systemic lupus erythematosus. Annals of the Rheumatic Diseases, 72 (Suppl 3), A827-A828.

Zhang, L., Su, Z., Zhang, Z., Lin, J., Li, D., \& Pflugfelder, S. (2015). Effects of azithromycin on gene expression profiles of proinflammatory and anti-inflammatory mediators in the eyelid margin and conjunctiva of patients with meibomian gland disease. Jama Ophthalmology, 133 (10), 1117-1123. doi:10.1001/jamaophthalmol.2015.2326

Zuo, E., Cai, Y., Li, K., Wei, Y., Wang, B., Sun, Y., . . . Yang, H. (2017). One-step generation of complete gene knockout mice and monkeys by CRISPR/Cas9-mediated gene editing with multiple sgRNAs. Cell research, 27 (7), 933-945. doi:10.1038/cr.2017.81

\section{Figure legends}

Figure 1 Effects of AZM on RA FLSs. (A) Following exposure to TNF- $\alpha$ and IL- $1 \beta$ for 6 h, RA FLSs $(\mathrm{n}=3)$ were treated by AZM or Vehicle for another $24 \mathrm{~h}$. IL-6 (A) , IL-8 (B), MMP-1(C) and MMP-3 (D) levels in the supernatant of RA FLSs were analysed by ELISA. The migration (E) and invasion(F) abilities were assessed in AZM or Vehicle treated RA FLSs from six different patients (3 males and 3 females). Data represent independent experiments performed in triplicate, and six different fields were selected for 
cell counting (graph below). RA FLSs passing through the polycarbonate membrane with ECM coating shows that cell invasion requires the ECM proteolysis step in addition to migration. RA FLSs ( $\mathrm{n}=3$ ) were treated by AZM or Vehicle for $24 \mathrm{~h}$, followed by TNF- $\alpha$ or IL-1 $\beta$ stimulation for $6 \mathrm{~h}$. CXCL9 (G), CXCL10 $(\mathbf{H})$ levels in the culture supernatant were measured by ELISA. (I)After treatment as indicated in (A-D), cell-free RA FLSs supernatant was collected and used as a chemo-tactic source for healthy donor peripheral blood leucocytes $(n=3)$ in a transwell migration system for $6 \mathrm{~h}$. The number of migrating leucocytes in the lower compartment was counted. ${ }^{*} P<0.05,{ }^{* *} P<0.01$ and ${ }^{* * *} P<0.001$ compared with Vehicle. (J) The production of VEGF decreased when RA FLSs were exposed to AZM.(K) Tube formation assay was applied to determine the angiogenic ability of HUVECs after treatment with conditioned medium of AZM or its Vehicle treated RA FLSs. Data in A-D, G and H are expressed as the mean of three samples \pm SD and represent three independent experiments. ${ }^{*} P<0.05$, ${ }^{* *} P<0.01$ and ${ }^{* * *} P<0.001$ compared with Control. IL-1 $\beta$, interleukin- $1 \beta \cdot$ TNF- $\alpha$, tumour necrosis factor- $\alpha \cdot$ AZM, azithromycin; Ctrl, control.

Figure 2 Anti-arthritis effects of AZM on CIA. Mice immunized with CII were randomly divided into 4 groups ( $\mathrm{n}=6$ mice for each group and time point) and administered AZM or Vehicle at the indicated doses twice a day after the initial immunization. The data are representative of four independent experiments with similar results. (A)Arthritis scores were monitored once per five days. (B) Hind paw thickness was calibrated from the 21st day following the first immunization. AZM (low): ${ }^{\#} P<0.05,{ }^{\#} P<0.01$, \#\#\# $P$ $<0.001$ compared with Vehicle. AZM (medium): ${ }^{*} P<0.05,{ }^{* *} P<0.01,{ }^{* * *} P<0.001$ compared with Vehicle. AZM (high): ${ }^{\&} P<0.05,{ }^{\& \&} P<0.01,{ }^{\& \& \&} P<0.001$ compared with Vehicle.(C) Fore paw (upper) and hind paw (lower) photographs obtained on day 42 from mice with CIA from the day of first immunization.(D) Representative histology images by H\&E staining about interphalangeal joint (upper) and ankle joint (lower) obtained on day 67 from mice with CIA with the indicated AZM treatment. Pathological changes, including synovial proliferation (red arrowhead) and joint destruction (yellow arrowhead), are shown. (E) Inflammation, hyperplasia, cartilage degradation and bone destruction were measured through a scoring system ( $\mathrm{n}=12$ mice per group). (F)Representative micro-CT images of hind paws and interphalangeal joints (red square). (G) BV/TV, Tb. BMD, Tb. Th, Tb. N and Tb. Sp in the distal tibia were assayed by micro-CT and 3D reconstruction. E and G: ns: not significant, ${ }^{*} P<0.05,{ }^{*} P<0.01$ and ${ }^{* * *} P<0.001$ compared with Vehicle group. CIA, collagen-induced arthritis; BV/TV, Bone volume fraction; Tb. BMD, trabecular bone mineral density; Tb. Th, trabecular thickness; Tb. N, trabecular number; Tb. Sp, trabecular separation.

Figure 3 Functional enrichment analysis of differentially expressed genes. (A) Hierarchical clustering of the dysregulated mRNAs in RA FLSs. The expression values were represented by a color scale. The intensity increased from blue (relatively lower expression) to red (relatively higher expression). Each column represented one tissue sample, and each row represented a single mRNA. (B) GO analyses of the host genes of differentially expressed mRNAs and GO annotations of the host genes of differentially expressed mRNAs. The bar plot presented the enrichment scores (-loge[p value]) of the top 10 significantly enriched GO terms in biological processes, cellular components and molecular functions. (C) KEGG analyses of the host genes of differentially expressed mRNAs and KEGG annotations of the host genes of differentially expressed mRNAs. The bubble diagram presented the enrichment scores (-loge[p value]) of the top 10 significantly enriched KEGG terms in molecular function and signal pathway. (D) After stimulation with AZM for 24 $\mathrm{h}$, total RNA was extracted from RA FLSs and subjected to qRT-PCR for the assessment of CHOP mRNA levels. qRT-PCR data are expressed as the mean \pm SD of six samples ( 3 males and 3 females) from two independent experiments. NS: not significant, ${ }^{*} P<0.05$ and ${ }^{* * *} P<0.001$ compared with vehicle. (E) Using similar treatments as (D), total and phosphorylated levels of PERK and elF2 $\alpha$, and the increased expression of IRE1 $\alpha$, ATF 4 were analysed by western blot. Western blot data are representative of two independent experiments from six different patients (3 male and 3 female) with similar results. (F) Under the stimulation of TNF- $\alpha$ and IL-1 $\beta$, similar treatment as (D) was used, total and phosphorylated levels of p38 and Akt, and the increased expression of CREB3L2 were analysed by western blot. Western blot data are representative of two independent experiments from six different patients ( 3 male and 3 female) with similar results. (G) RA FLSs $(\mathrm{n}=3)$ were treated with AZM or its vehicle for $24 \mathrm{~h}$, Annexin X staining were applied to detect the cell apoptosis of RA FLSs $(\mathrm{n}=3)$. The statistical significance of differences between AZM 
and vehicle-treated groups was determined. Data represent the mean \pm SD of three independent replicates of three samples. ${ }^{* *} P<0.01$ and ${ }^{* * *} P<0.001$ compared with Control. DMSO, Dimethyl Sulfoxide; Tm, tunicamycin; Tg, thapsigargin.

Figure 4 GRP78 is a novel target of AZM. (A) Coomassie blue staining of DARTS assay. The band with molecular weight around $80 \mathrm{kDa}$ protected by AZM was indicated by arrow. (B) DARTS and Western blot to confirm AZM's binding targets. (C and D) CETSA melt response. (E) DARTS assay with serial deletion constructs encoding Flag-tagged mutants of GRP78. GRP78 (aa 1-750), GRP78 (aa 126-750), GRP78 (aa 406-750) and GRP78 (aa 1-479). 293T cells were transfected with Flag tagged mutants of GRP78 plasmids, as indicated. DARTS assay samples were detected by Flag antibody. (F)Potential targeting sites of AZM towards GRP78. (G) DARTS assay for Asp-178 point mutant of cPLA2. The Asp-178 of cPLA2 was substituted with Ala505. 293T cells were transfected with the point mutant plasmid and DARTS was performed. Point mutated cPLA2 was detected by Flag antibody. $(\mathbf{H})$ The double reciprocal diagram of ATP concentration and luminescence intensity under different concentrations of AZM showed that the treatment of AZM might be related to ATP.(I) The enzyme activity of GRP78 was measured in the presence of AZM or HA15. The initial concentration of Pronase was $10 \mathrm{mg} / \mathrm{kg}$. ns: not significant, ${ }^{* *} P<0.01$ and ${ }^{* * *} P<0.001$ compared with Vehicle. Temp, Temperature.

Figure 5 AZM inhibits GRP78 activity through binding to its catalytic domain. (A) RA FLSs were stimulated with AZM for $24 \mathrm{~h}$, immunoprecipitation and immunoblotting were performed on total protein with anti-GRP78 or anti- PERK, IRE1 $\alpha$ or ATF6 $\alpha$ antibodies. (B)Equal aliquots of nuclear and membrane protein from AZM or its vehicle treated RA FLSs with GRP78 overexpression or not were pooled (total, $30 \mathrm{mg}$ ) and subjected to SDS-PAGE and immunoblot analysis for the indicated protein. A and B: The results are representative of four samples (2 male and 2 female) from independent experiment.

Figure 6 AZM activates UPR and promote apoptosis via GRP78. (A)GRP78 expression in Hela cells when GRP78 was knocked out using CRISPR-Cas9 technique. (B) Total and phosphorylated levels of PERK, elF2 $\alpha$ and IRE1 $\alpha$, the expression of ATF6 $\alpha$ (p50), ATF4, CHOP, as well as the expression of m-SERBP-1c in membrane or nuclear compartments, were detected in RA FLSs with GRP78 deficiency or not. $\beta$-Actin was used as a loading control. (C) Silencing efficiency of siRNA targeting GRP78 (siGRP78) was detected by western blot. The two siRNAs were combined at equal concentrations for the subsequent experiments. The negative control siRNA is referred to as siCtrl. The results are representative of two independent experiments with three different samples in each. (D) Following exposure to TNF- $\alpha$ and IL-1 $\beta$ for $6 \mathrm{~h}$, RA FLSs with GRP78 knocking down or not were treated by AZM or Vehicle for another $24 \mathrm{~h}$. IL-6, IL-8, MMP-1, MMP-3, CXCL9 and CXCL10 levels in the supernatant of RA FLSs were analysed by ELISA. The data are expressed as the mean $\pm \mathrm{SD}$ of three samples and are representative of three independent experiments. ${ }^{* * *} P<0.001$ compared with SiCtrl. (E) RA FLSs with GRP78 silencing or not were treated with AZM or its vehicle for $24 \mathrm{~h}$, Annexin X staining were applied to detect the cell apoptosis of RA FLSs $(\mathrm{n}=3) .{ }^{* * *} P<0.001$ compared with SiCtrl. GRP78 ${ }^{\mathrm{wt}}$, GRP78 wild type. GRP78 ${ }^{-/-}$, GRP78 deficiency. GRP78 $^{\mathrm{mt}}$, GRP78 mutation type.

Supplementary Figure 1 Effects of Etanercept and AZM on the production of pro-inflammatory factors. Following exposure to TNF- $\alpha$ and IL-1 $\beta$ for 6 h, RA FLSs were treated by AZM, Etanercept or in combinaton for another $24 \mathrm{~h}$. IL-6 (A), IL-8 (B) , MMP-1(C) and MMP-3 (D) levels in the supernatant of RA FLSs $(\mathrm{n}=3$ ) were analysed by ELISA. Data are expressed as the mean $\pm \mathrm{SD}$ of three samples and are representative of three independent experiments. ns: not significant, ${ }^{*} P<0.05,{ }^{* *} P<0.01$ and ${ }^{* * *} P<0.001$ compared with Vehicle group. ETC, etanercept.

Supplementary Figure 2 Effects of AZM on the production of pro-inflammatory factors in PBMC. Following exposure to TNF- $\alpha$ and IL- $1 \beta$ for 6 h, RA FLSs were treated by AZM, Etanercept or in combination for another $24 \mathrm{~h}$. IL-6 (A), IL-8 (B), TNF- $\alpha$ (C), IL-1 $\alpha$ (D) and IL-1 $\beta$ (E) levels in the supernatant of RA FLSs were analysed by ELISA. Data are expressed as the mean \pm SD of six samples (3 male and 3 female) and are representative of three independent experiments. ns: not significant, ${ }^{*} P<0.05$, ${ }^{* *} P<0.01$ and ${ }^{* * *} P<0.001$ compared with Vehicle group. 
Supplementary Figure 3 Effects of AZM on cytokine production in CIA models. The serum from AZM or Vehicle treated CIA mice ( $\mathrm{n}=6$ mice per group) were collected for ELISA detection of IL-1 $\beta$ and IL-6, COMP, IL-10, IL-13, OPG and RANKL on the 42nd day after the first immunization. This experiment data is representative of two independent experiments from 6 mice per group. ${ }^{* *} P<0.01$ and ${ }^{* * *} P<0.001$ compared with Vehicle in the Control or CIA group.

Supplementary Figure 4 qRT-PCR validation of fourteen differentially expressed mRNAs in 20 pairs of RA FLSs samples. The relative expression levels of 11 up-regulated and 3 down-regulated mRNAs (selected from the top 10 dysregulated mRNAs) in 20 pairs of AZM and Vehicle treated RA FLSs. ns: not significant, ${ }^{*} P<0.05,{ }^{* *} P<0.01$ and ${ }^{* * *} P<0.001$ compared with vehicle.

Supplementary Table 1 Clinical characteristics of RA patients.

Supplementary Table 2 Primer sequences of genes in real-time fluorescent quantitative PCR (qRT-PCR).

Supplementary Table 3 List of potential AZM targets in RA FLSs identified by mass spectrometry.

Supplementary Table 4 Primer sequences of sgGRP78 in CRISPR/Cas9. 
Figure 1

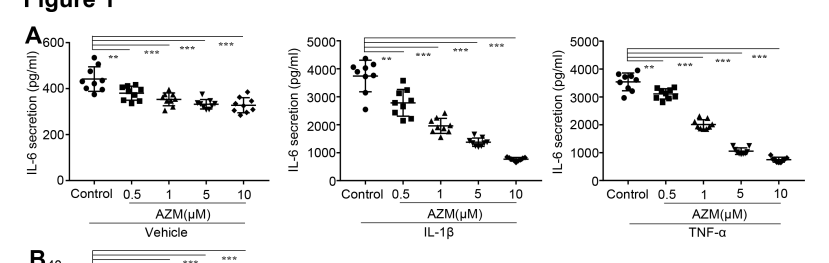

和和事
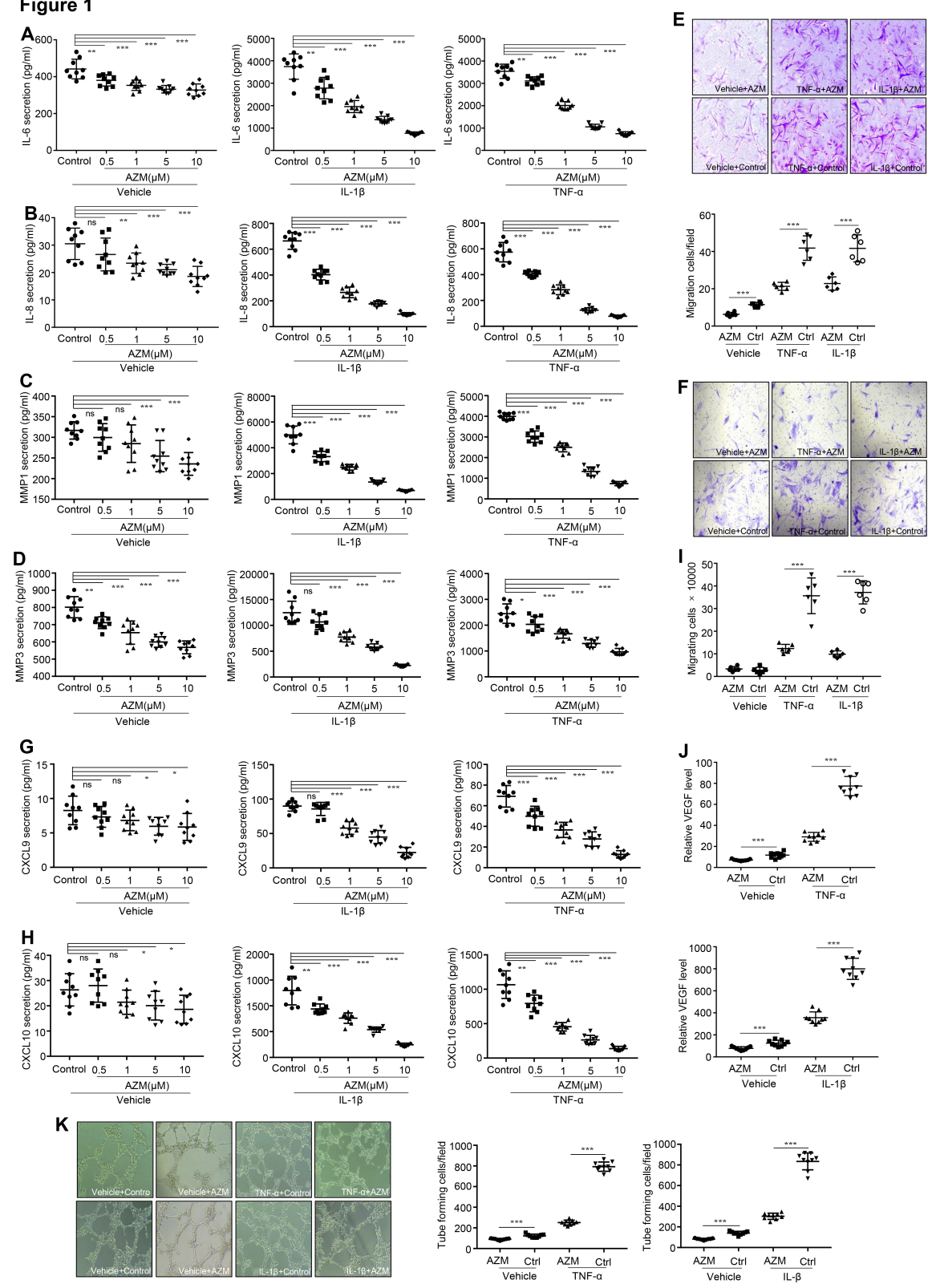

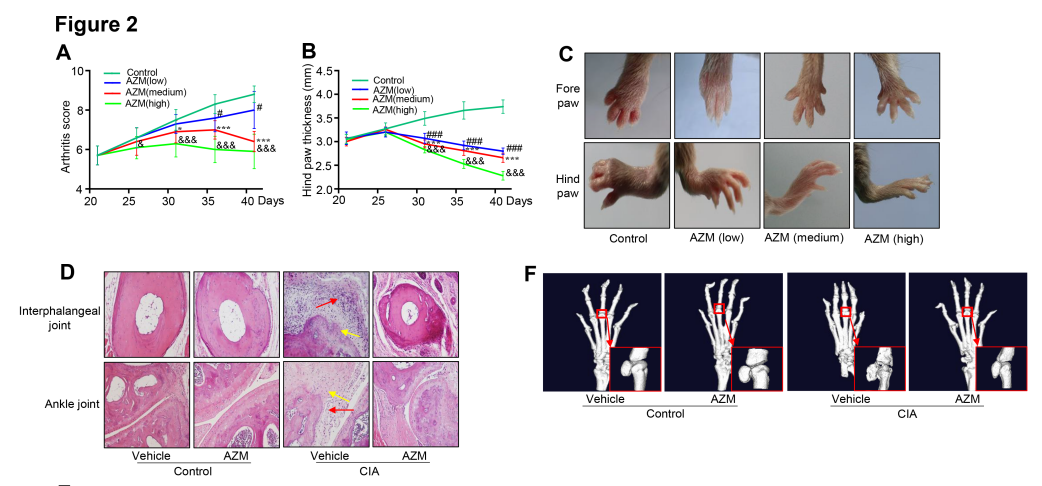

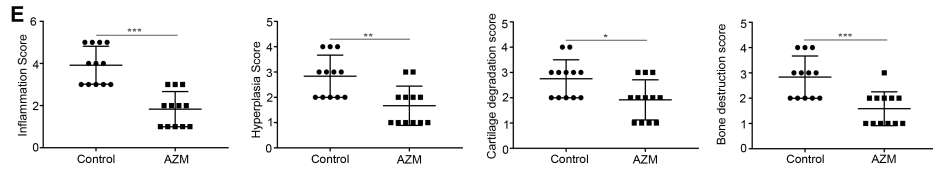

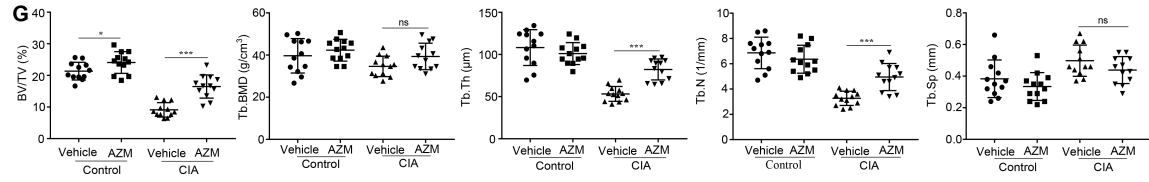




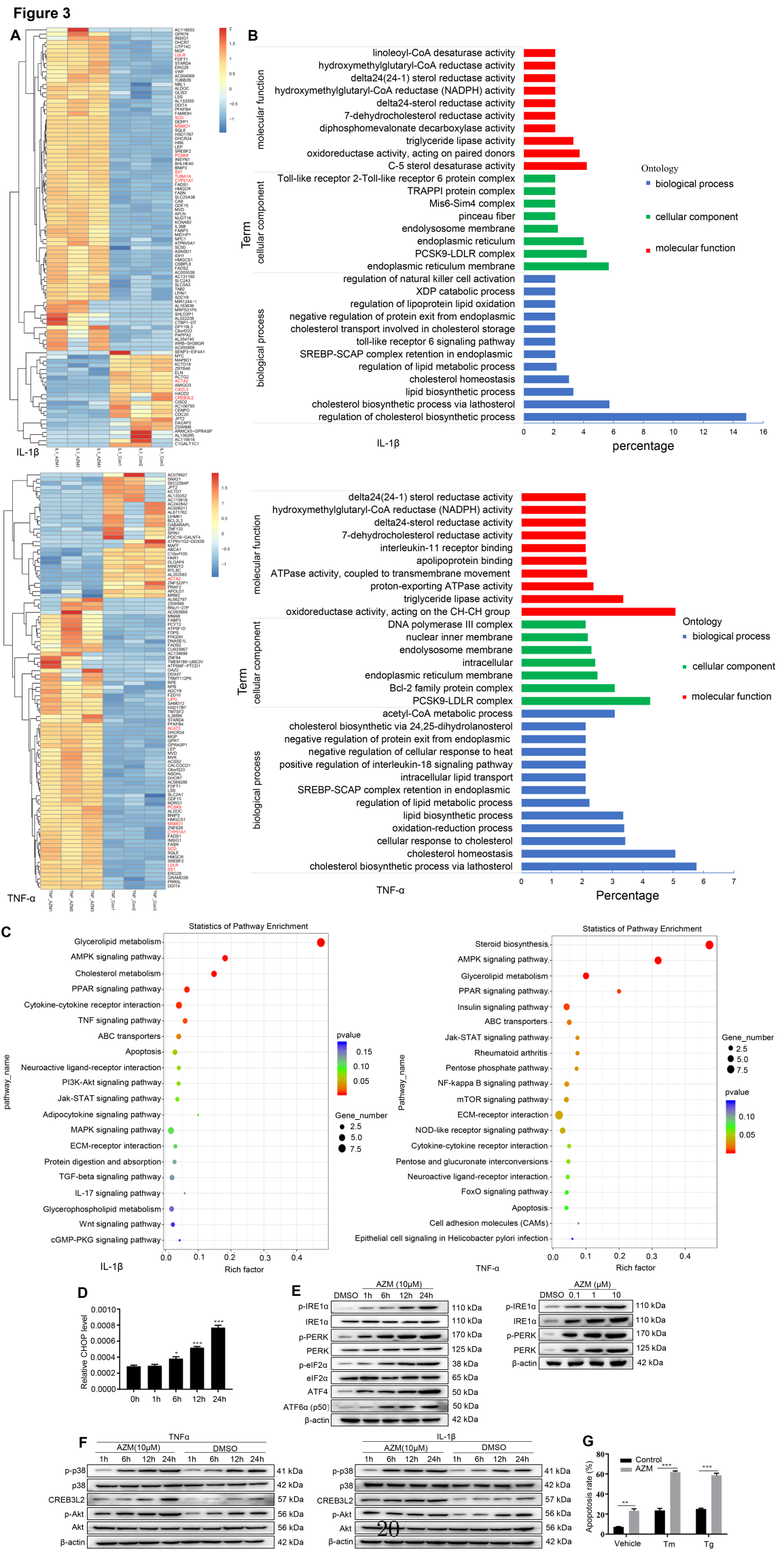



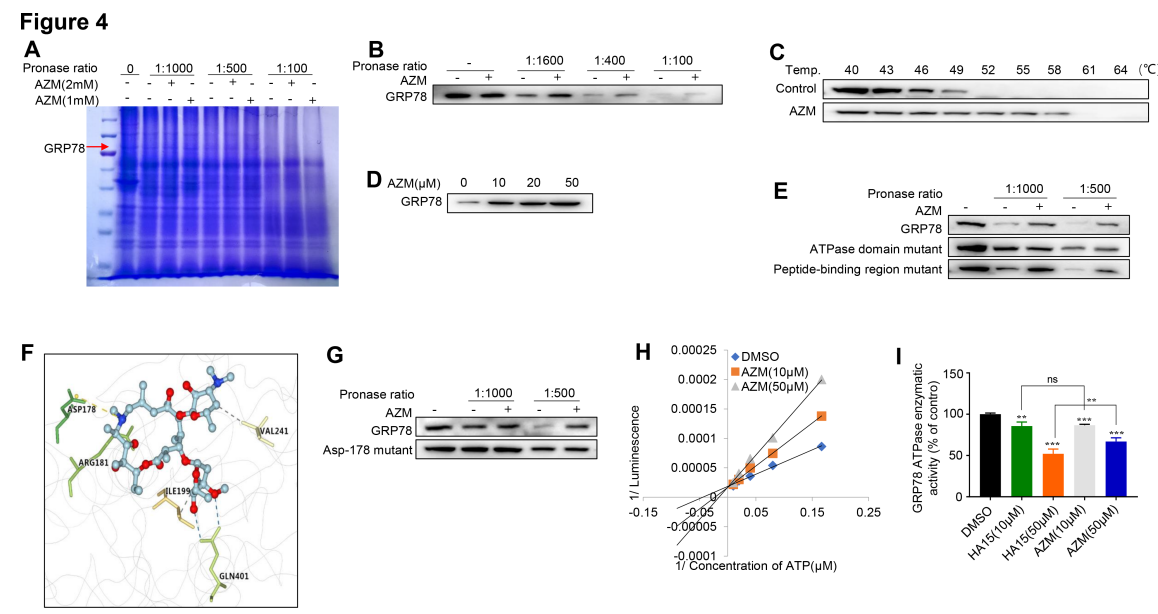

\section{Figure 5}

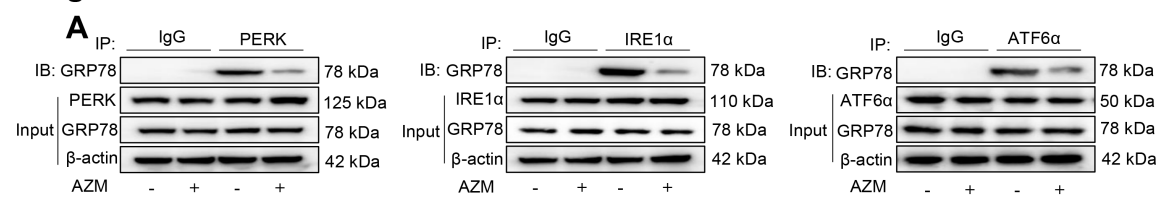

B

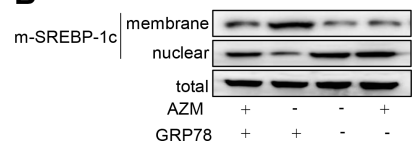


Figure 6
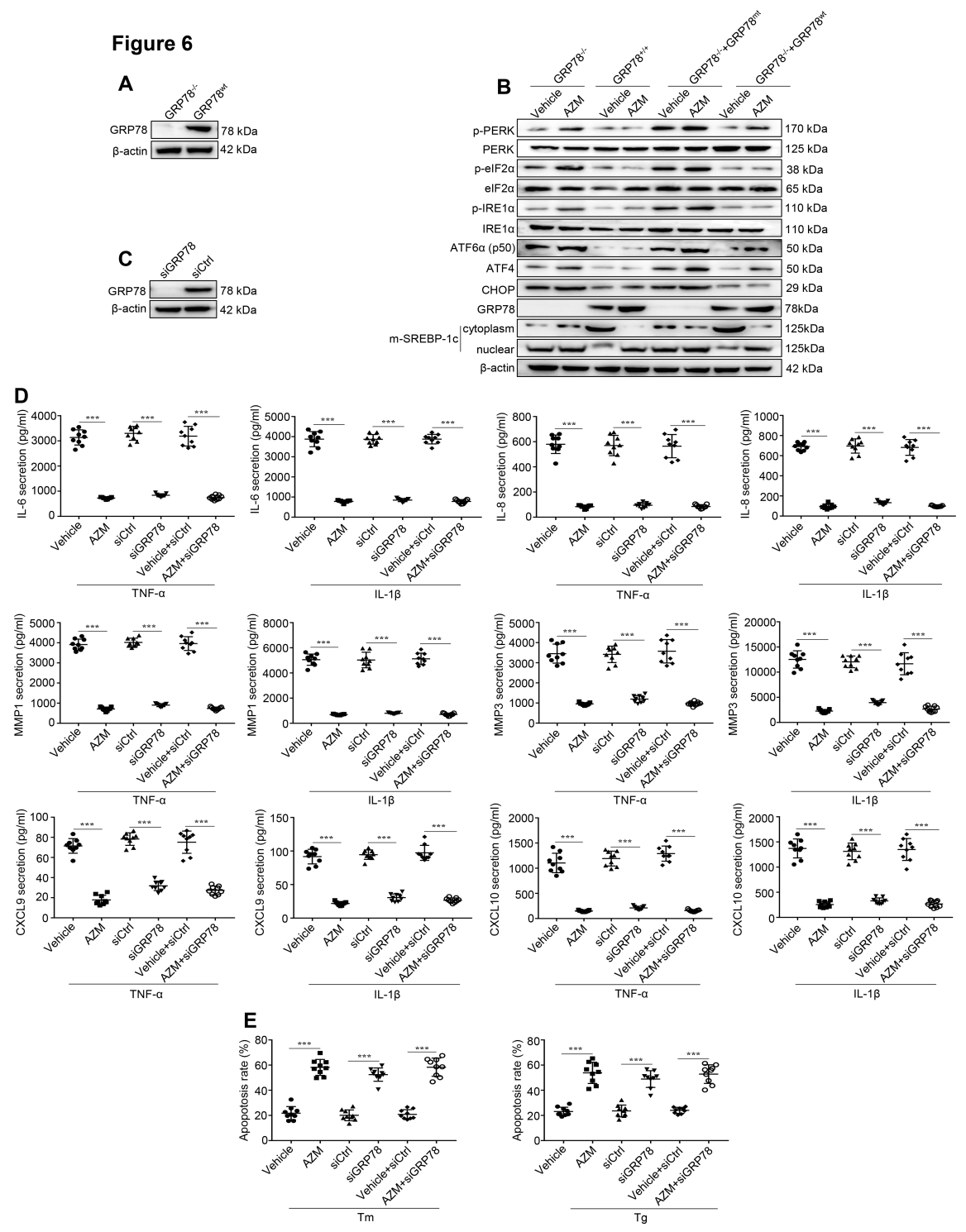

Supplementary Figure 1
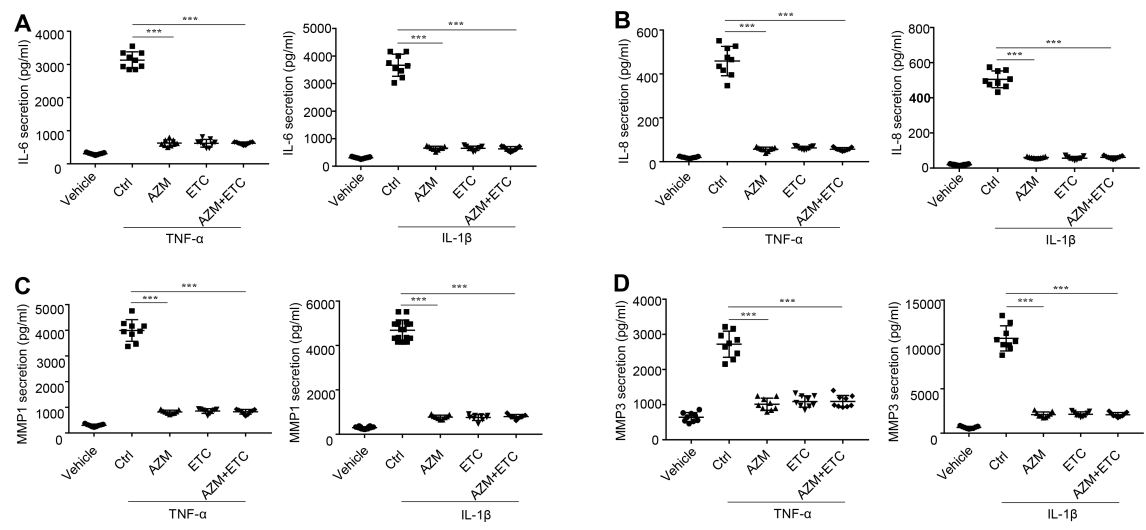
Supplementary Figure 2
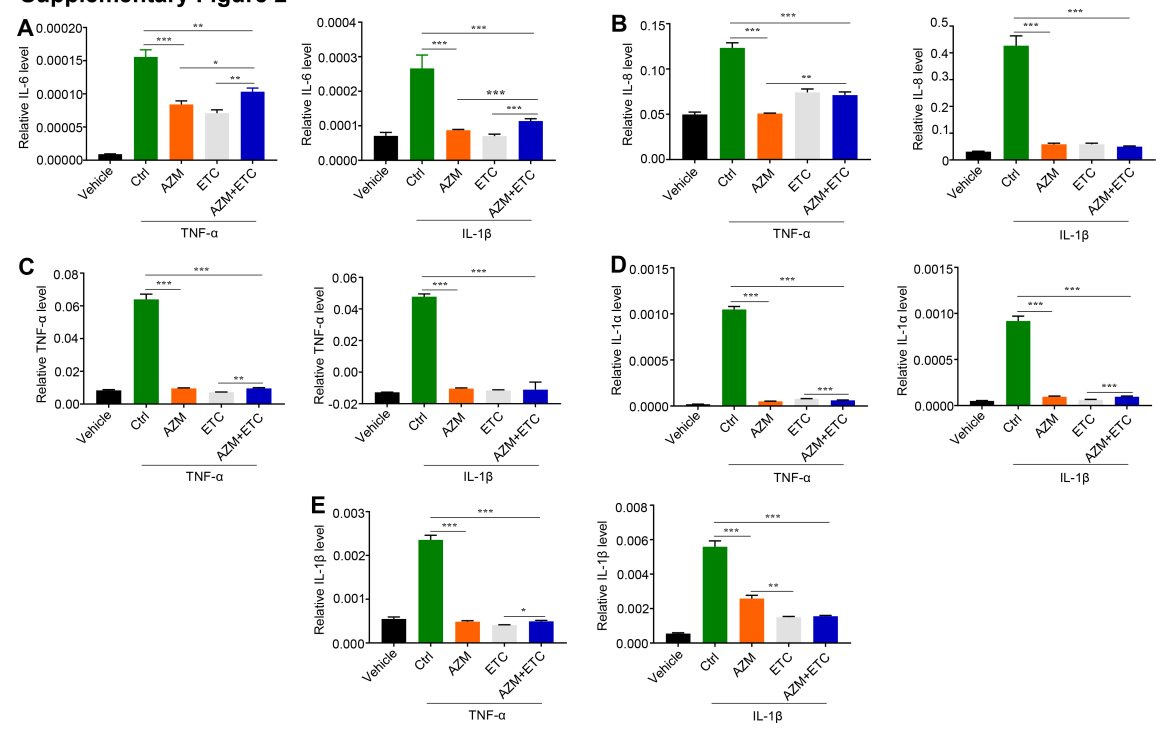

\section{Supplementary Figure 3}
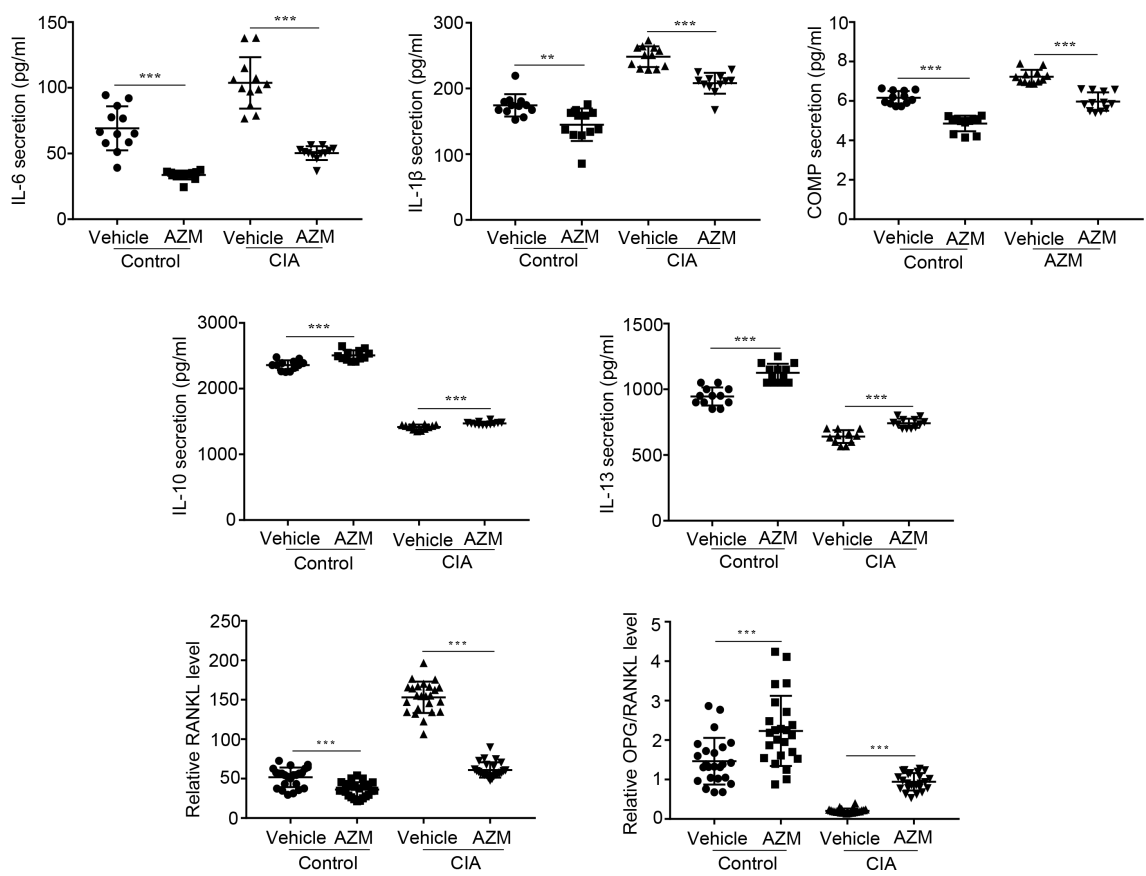


\section{Supplementary Figure 4}
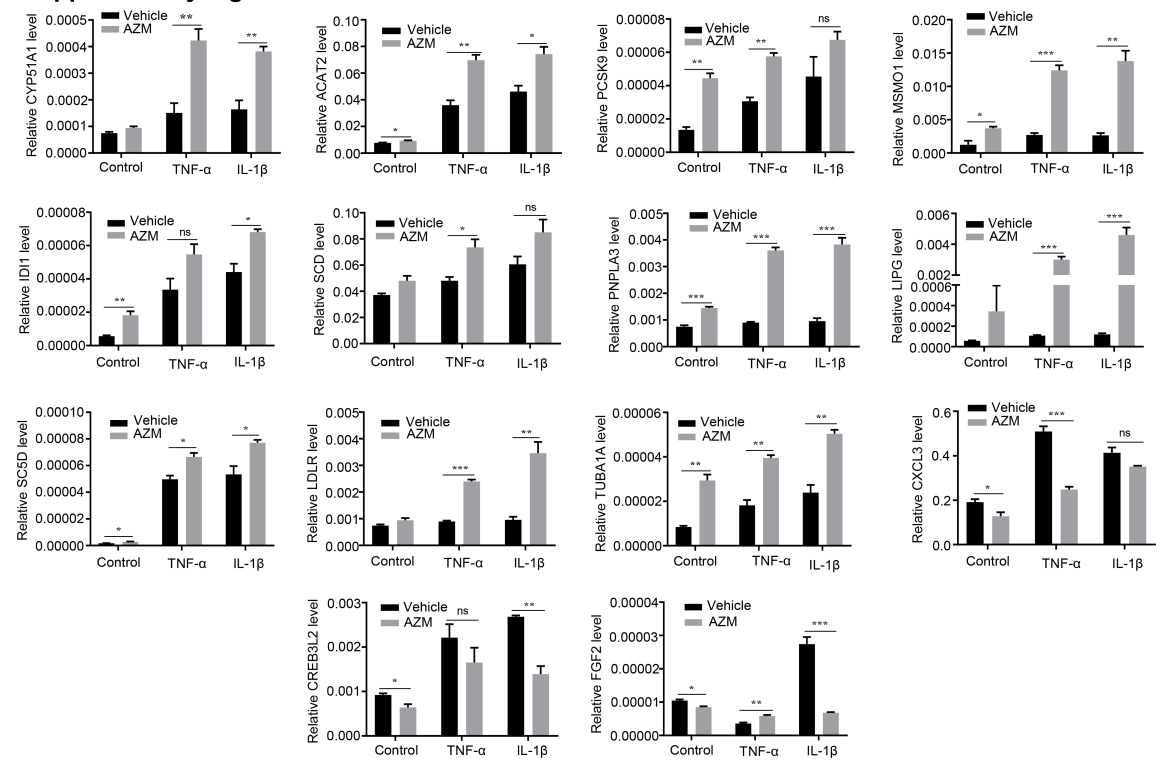

\section{Hosted file}

Supplementary Table 1.pdf available at https://authorea.com/users/390677/articles/504927azithromycin-alleviates-the-severity-of-rheumatoid-arthritis-via-targeting-uprcomponent-grp78

\section{Hosted file}

Supplementary Table 2.pdf available at https://authorea.com/users/390677/articles/504927azithromycin-alleviates-the-severity-of-rheumatoid-arthritis-via-targeting-uprcomponent-grp78

\section{Hosted file}

Supplementary Table 3.pdf available at https://authorea.com/users/390677/articles/504927azithromycin-alleviates-the-severity-of-rheumatoid-arthritis-via-targeting-uprcomponent-grp78

\section{Hosted file}

Supplementary Table 4.pdf available at https://authorea.com/users/390677/articles/504927azithromycin-alleviates-the-severity-of-rheumatoid-arthritis-via-targeting-uprcomponent-grp78 